\title{
Opportunities and Challenges for Error Correction Scheme for Wireless Body Area Network-A Survey
}

\author{
Rajan Kadel ${ }^{1, *}$, Nahina Islam ${ }^{1}\left[\right.$, Khandakar Ahmed ${ }^{2} \mathbb{D}$ and Sharly J. Halder ${ }^{1}$ \\ 1 Melbourne Institute of Technology, School of IT and Engineering (SITE), Melbourne 3000, Australia; \\ nislam@mit.edu.au (N.I.); sjhalder@mit.edu.au (S.J.H.) \\ 2 College of Engineering and Science, Victoria University, Footscray 3011, Australia; \\ khandakar.e.ahmed@ieee.org \\ * Correspondence: rkadel@mit.edu.au; Tel.: +61-3-8600-6745
}

Received: 5 November 2018; Accepted: 18 December 2018; Published: 23 December 2018

\begin{abstract}
This paper offers a review of different types of Error Correction Scheme (ECS) used in communication systems in general, which is followed by a summary of the IEEE standard for Wireless Body Area Network (WBAN). The possible types of channels and network models for WBAN are presented that are crucial to the design and implementation of ECS. Following that, a literature review on the proposed ECSs for WBAN is conducted based on different aspects. One aspect of the review is to examine what type of parameters are considered during the research work. The second aspect of the review is to analyse how the reliability is measured and whether the research works consider the different types of reliability and delay requirement for different data types or not. The review indicates that the current literatures do not utilize the constraints that are faced by WBAN nodes during ECS design. Subsequently, we put forward future research challenges and opportunities on ECS design and the implementation for WBAN when considering computational complexity and the energy-constrained nature of nodes.
\end{abstract}

Keywords: automatic repeat request (ARQ); error correction; hybrid automatic repeat request (HARQ); wireless body area network (WBAN)

\section{Introduction}

Wireless body area networks (WBANs) is termed as a wireless network in and around the human body. This network combines the study of medical and communication technologies. WBAN has received increased attentions and it has been widely researched due to its possible use on medical and non-medical applications. WBAN is an essential process for many applications, such as medical sensing and control, health and wellness nursing, personal audio or video, wearable computing, location finding, and identification. As WBAN is around a human body, the communication distance between devices in WBAN cover only a short range, typically a few meters [1-5].

WBANs come with opportunities as well as challenges. It is anticipated that wearable and implant sensors used in WBAN will facilitate advanced way for human wellbeing monitoring [6]. These opportunities come with various challenges. Firstly, power consumptions on implant and wearable sensors should be ultra-low to boost network's life, as these devices are battery operated and battery replacement is a complex medical procedure for certain conditions $[1,4]$. Secondly, the network is required to handle various types of data, including medical and non-medical. The expected Quality of Service (QoS) and delay requirement for those data are different. In addition, the network should provide ways to handle different types of data and delay requirements for those data. Finally, the interference that is caused by the existence of other networks should be considered during WBAN implementation [7]. 
The design and implementation of Error Correction Schemes (ECSs) in WBAN have numerous challenges. First, some nodes in WBAN are energetically and computationally constrained as the operation of node is performed by battery and battery replacement is a complex process. Second, the ECS used in the WBAN should be able to support different levels of reliability according to data (medical and non-medical data) and variable data rates.

The proper design and implementation of ECS on WBAN may lead to energy saving on the WBAN nodes as well as improving the reliability of the communication link in WBAN. The energy saving on WBAN nodes, such as implant and wearable nodes, are very important. The energy saving at energy-constrained node may lead to better quality of life as it helps to reduce the number of complex medical procedure. In addition, health and wellness monitoring of human can be enhanced significantly, as we would be able to improve the reliability of WBAN communication using ECS. Therefore, the proper understanding of ECS on WBAN will help to improve the reliability of communication links on WBAN and support different reliability for different sets of data. The motivation of this paper is to present the current scenarios of ECS on WBAN and future opportunities and challenges regarding ECS design and the implementation of WBAN.

In this paper, we provide a brief overview of ECS and WBAN, including IEEE standard, channel and network models, followed by a detailed literature review on the proposed ECS for WBAN. The review is conducted based on different aspects. The first aspect of the review is to examine the parameters that are considered during the research work by the researcher. The key parameters for the study or experiment are the type of WBANs, environment of the network, IEEE standard, and physical media. Firstly, the study can consider the network with one hop or two-hop extended star network. Secondly, the study can be conducted using standalone WBAN only or WBAN with a certain environment. Thirdly, the study may depend on the IEEE standard and the physical media i.e., frequency band.

The second aspect of the review is whether the research work considers different types of reliability and delay requirement for different data types (medical and non-medical data) or not. Another key review area is how the reliability of WBAN is measured and what type of data (random or real data) is used during the research work. The reliability of a network can be measured in various units, such as Bit Error Rate (BER), Packet Error Rate (PER), Packet Acceptance Rate (PAR), Received Signal Strength (RSS), throughput efficiency, Mean Absolute Error (MAE), and residual BER. We make two major contributions in this paper. First, we conducted an extensive review on available literature on ECS on WBAN. Second, we also put forward future research challenges and opportunities on design and implementation of ECS on WBAN.

The remainder of the paper is organized as follows. In Section 2, we outline the various types of ECS that are available in the literature that are used in communication systems. Section 3 summaries IEEE 802.15.6 standard for WBAN, which is followed by channel and network models in Section 4 . In Section 5, we review papers on different aspects. Section 6 presents future research issues, including unsolved challenges in WBAN. Finally, we present concluding remarks of the paper in Section 7. All of the acronyms used in this paper are presented in Table A1 in the Appendix Section.

\section{Error Correction Scheme (ECS)}

In the literature, there are various types of ECS presented to support various types of reliability, delay constrained, and data rates in different types of communication systems. Here, we present a brief overview of various types of ECS used in the communication. There are several types of ECSs available to maintain and enhance the reliability and delay-constraints of a communication link. These ECSs were loosely grouped in to four categories. Figure 1 illustrates a broad classification of ECS for WBAN error correction context. 


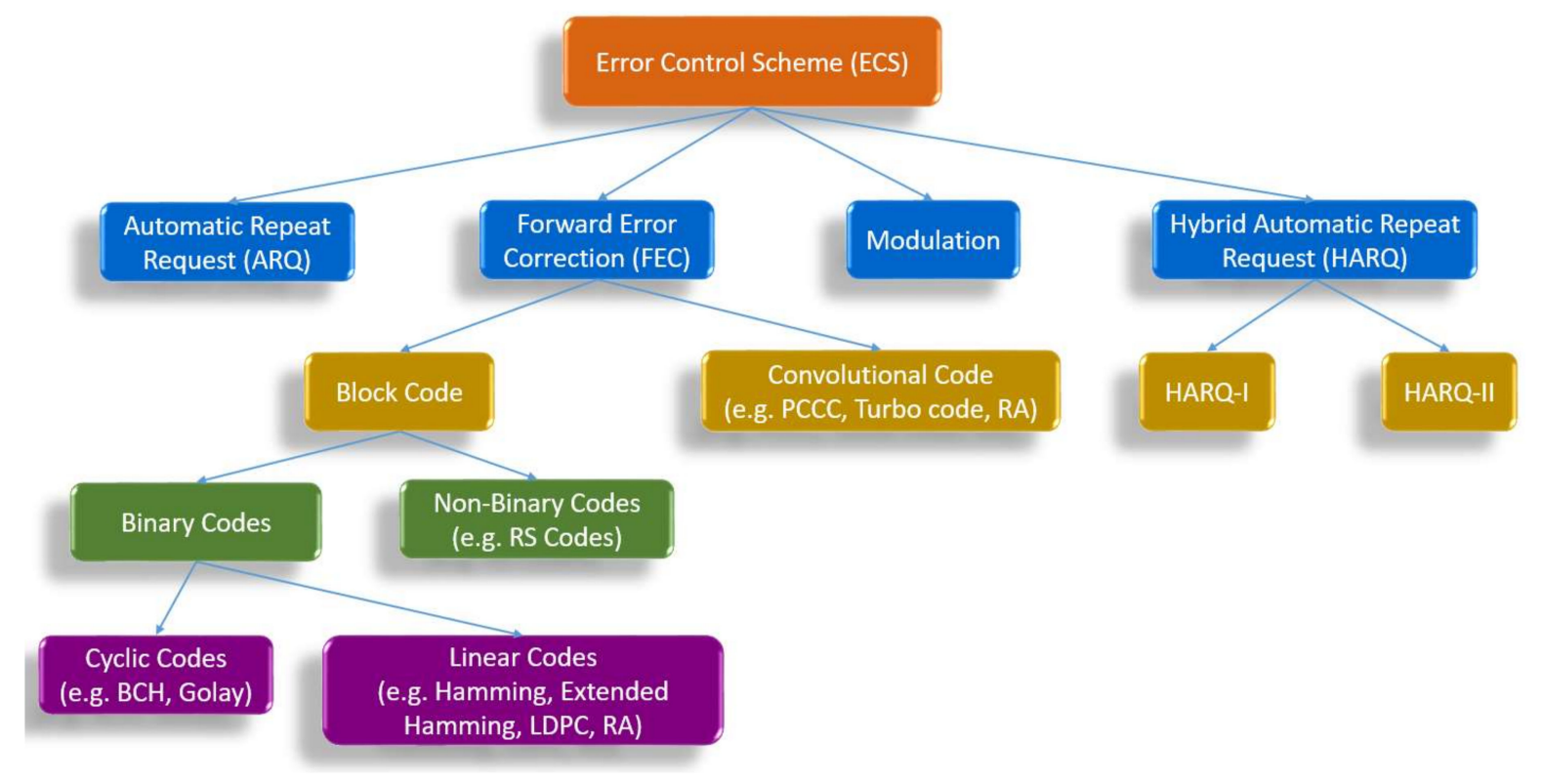

Figure 1. Classification of Error Correction Schemes (ECS) for wireless body area networks (WBAN) error correction context.

\subsection{Automatic Repeat Request (ARQ)}

In automatic repeat request (ARQ) [8], transmitter retransmits the packet if the receiver found errors. The error-detection at the receiver is usually performed by using error-detection techniques, such as parity checking and cyclic redundancy check (CRC). Until the receiver received the error-free packet, the retransmission process is continued. In this process, the transmitter applied the error-detecting code to the packet before transmission. Afterwards, the checksum is calculated at the receiver to find whether error has occurred or not. If the checksum is not equal to right value, the ARQ scheme send negative acknowledgement (NACK) to the transmitter while using the feedback channel and the transmitter resend packet. Otherwise, the receiver send positive acknowledge (ACK). In this scheme, the transmitter resends the packet if it does not received ACK packet within a pre-specified timeframe. The main downside of this scheme is the additional cost of retransmission. The retransmission is very common when channel quality is poor and rarely happens when the channel quality is good. ARQ scheme consist of error detection and re-transmission and it involves two bottom layers (Physical (PHY) layers and Media Access (MAC) Layer) of the Open Systems Interconnection (OSI) model.

\subsection{Forward Error Correction (FEC)}

Forward error correction (FEC) [9] or channel coding [10] is a classical approach that is used to enhance the performance of communication link. FEC is used in communication when retransmission is relatively very costly and delay sensitive. In FEC, Error-Correcting Codes (ECCs) add redundancy to the transmitted packet at the transmitter side that allows for the detection and correction of a certain amount of error at the receiver side. The error can be detected and corrected at the receiver. Hence, it eliminated the need of retransmission that is the key advantage of FEC. The introduction of FEC allows a system to operate at a significantly lower Signal to Noise Ratio (SNR) than an uncoded system to achieve a certain BER. The difference in SNR is called coding gain due to FEC and the coding gain depends on the type of ECC and the decoding algorithm and complexity. The complexity of algorithm determines the decoding power consumption. However, the lower requirement of transmission power at the transmitter comes with extra costs: energy consumption due to encoding at the transmitter, decoding at the receiver, and the transmission of extra bits introduced by FEC due to redundancy. Therefore, the use of FEC is only justified if extra power consumption introduced due to encoding, 
decoding, and transmission of extra bits is lower than the power saving due to the use of FEC in WBAN because of energy-constrained at the node.

There are different types of FEC available in the literature with varying complexity and performance. ECCs is classified into two types: block codes and convolutional codes [11]. The discussion of block codes here is limited to binary block codes from the binary Galois Field $G F(2)$ and the concept can be generalized for the non-binary field. In block codes, the message to be transmitted is divided into smaller blocks of pre-defined length. Subsequently, these blocks are encoded into codewords using encoder. Commonly, a block code is represented by the triple $(n, k, t)$, where $n$ is the length of code word bits, $k$ is the length of information bits in the code word, and $t$ is the error correction capability in term of number of bits that can be corrected. Figure 2 illustrates information bits and parity bits in block codes. The code rate $(R)$ of ECC is defined as Equation (1) [11].

$$
R=\frac{k}{n}
$$

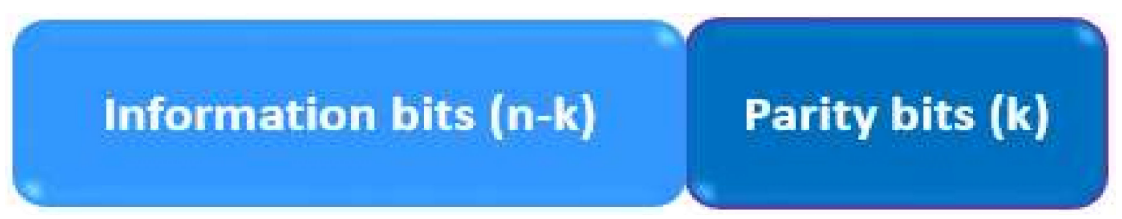

Figure 2. Illustration of Block Code with codeword length $n$.

Let $E_{b} / N_{0}$ be defined as the ratio of energy per bit $\left(E_{b}\right)$ to the spectral noise $\left(N_{0}\right)$ and $E s / N_{0}$ is defined as the ratio of energy per symbol $\left(E_{s}\right)$ to the spectral noise over the Additive White Gaussian Noise (AWGN) channel. Afterwards, the relationship between $E s / N_{0}$ and $E_{b} / N_{0}[11,12]$, both expressed in $\mathrm{dB}$, as in Equation (2).

$$
\frac{E_{b}}{N_{0}}=\frac{E_{s}}{N_{0}}-10 \log _{10}(I)
$$

where $I$ is the number of information bits per symbol depends on the size of the modulation alphabet $(M)$ and the code rate of an ECC. The number of information bits per symbol is the product of the code rate and the number of coded bits $(M)$ per modulated symbol $[11,12]$ and is written as in Equation (3).

$$
I=R \log _{2}(M)
$$

For binary modulation (i.e., $M=2$ ), $I$ is equal to $R$. Subsequently, Equation (2) only depends on the code rate of ECCs. The code rate for the uncoded system is equal to one i.e., $R=1$ and the code rate for coded system is less than one i.e., $R<1$. To make fair comparison between coded and uncoded system, it needs to consider energy consumption due to Radio Frequency (RF) transmission of extra bits in coded system, encoding and decoding cost during result comparison. The energy consumption due to encoding at the transmitter usually insignificant in many cases [13]. Consequently, the main factors that should be considered are the decoding cost at the receiver (which depends on the type of decoding algorithm) and the transmission of extra bits due to redundancy that is introduced by FEC. Therefore, the comparison result between the coded and uncoded system should consider power per decoded bit, as well as the decoding cost of decoding algorithm.

There are different types of binary block codes available in the literature. A binary block code is called linear if and only if the modulo-2 sum of the two codes produces a new codeword. Hamming codes [14] and extended Hamming codes are categorized into linear binary block codes. Low-Density Parity Check (LDPC) [15] and Repeat Accumulate (RA) [16] are also linear block codes and these codes are considered as the most powerful codes exist in current literature. Another special class of the linear block code is cyclic code where a circular shift of a code word results in a new codeword. 
Bose-Chaudhuri-Hocquenghem (BCH) $[17,18]$ and Reed-Solomon (RS) [19] are categorized in to binary and non-binary cyclic codes, respectively.

Convolutional code is another category of FEC and it differs from block codes in many ways. First, the entire data stream isencoded into a single code word in convolutional code. Secondly, the encoder output $n$ at any given time not only depends on $k$ inputs but also $L$ previous input blocks i.e., constraint length in an $(n, k, L)$ convolution code. However, the code rate of convolutional code is defined the same as in block codes. There are various types of convolutional code and two common examples of convolutional codes are Parallel-Concatenated Convolutional Code (PCCC) [20], turbo code [21], and RA code [16].

The encoding and decoding complexity depends on the type of code and the decoding algorithm used. The encoding complexity of block code is very complex with larger values of information bits when the code is not linear. The encoding complexity of block code significantly reduces with the use of linear block codes. The cyclic codes have special algebraic properties that make encoding easy and decoding implementation using components, such as exclusive-OR gates, switches, and shift registers. The implementation of encoder of convolution code is very simple and can be performed using shift registers. Normally, the encoding operation is much simpler than the decoding operation. Thus, the encoder consumes substantially less power than the decoder does and the power that is consumed by the encoder can be ignored during the system evaluation.

There are several types of decoding algorithms existing in literature and there is a tradeoff between coding gain and the complexity of decoding. The decoding algorithms can be categorized depending on different factors: codes, decoding operations, input from the channels, etc. The decoding of short block codes, such as Hamming codes, can be performed by various methods: (a) syndrome decoding [22], (b) maximum likelihood (ML) decoding [23] either using decoding to the nearest code word or Viterbi algorithm [24] using trellis, and (c) maximum a posteriori (MAP) decoding with the BCJR algorithm [25]. A complex polynomial solver is to find error position during decoding of cyclic codes, such as $\mathrm{BCH}$ and RS codes. Viterbi decoding using trellis or MAP decoding or sequential decoding can be used for the decoding of convolutional codes.

Decoding algorithms can be categorized according to decoding operations into iterative decoding and non-iterative decoding. Syndrome decoding and ML decoding using the nearest code word for short block codes, algebraic decoding used in RS and $\mathrm{BCH}$ codes, and Viterbi decoding and sequential decoding for convolutional codes lie in non-iterative decoding category. MAP decoding with the BCJR algorithm that is used in Turbo codes and the sum-product algorithm (SPA) or belief propagation (BP) [26] that is used in LDPCs are categorized in to iterative decoding. The iterative decoding was further classified depending on the input to the decoding algorithms. If the decoder received the hard decision from the channel and the hard decision information used during the decoding iteration, then the decoder is referred to as hard-decision decoder. Whereas, if the decoder received the soft decision from the channel and the soft decision information used during the decoding iteration, then the decoder is referred to as soft-decision decoder. Soft decision decoder has better performance (i.e., coding gain) than the respective hard decision decoder. However, the soft decision decoder has higher complexity than the respective hard decision decoder. Binary message passing (BMP) decoding or Weighted Bit Flipping (WBF) is the hard decision version of SPA. The SPA also has lower complexity versions, called min-sum decoding [27]. The FEC scheme involves PHY layer of OSI model.

\subsection{Hybrid Automatic Repeat Request (HARQ)}

The performance of FEC is better during poor channel condition, whereas redundant bits that are introduced in FEC become an unwanted cost during good channel condition. The ARQ maintained reliable communication using retransmission that is very costly during poor channel due to frequent retransmission. However, the performance of ARQ is good during the good channel condition. Hybrid Automatic Repeat Request (HARQ) [28] scheme is developed by exploiting the advantages of both 
ARQ and FEC. In HARQ, the receiver tries to correct the detected errors and ask for retransmission request if error correction is not possible.

There are two types of HRAQ, called HARQ-I and HARQ-II. In the HARQ-I scheme, transmitter transmits coded packet and the coded packet retransmits if the receiver is unable to correct all errors during the decoding. HARQ-II is more complex than the HARQ-I. In HARQ-II scheme, the transmitter transmits coded packet with limited parity bits initially. If the receiver is unable to correct all errors, the transmitter transmits extra parity than the previously transmitted. Subsequently, the decoder attempts to decode by combining the previously received coded packet and extra parity bits at the receiver. This scheme is more bandwidth efficient than HARQ-I, but the HARQ-II scheme required storing a previously transmitted packet, but HARQ-I does not. As we discussed earlier, the HARQ scheme consists of both error detection and re-transmission, and thus involves both PHY and MAC layers of the OSI model.

\subsection{Modulation}

Another approach used in the literature other than pure error correction technique is adapting the modulation order according to channel condition. In this approach, the order of modulation is altered according to the quality of a communication link. When the quality of a communication channel is better i.e., better SNR, use higher order modulation, whereas the modulation order is decreased when the quality of communication link is poor to achieve the same BER [29]. Modulation scheme involves the PHY layer of the OSI model.

In the literature, we found that there are several types of ECSs that have been proposed to enhance communication reliability, delay constrained, and data rates in WBAN. In February 2012, IEEE 802.15.6 [30] is published as a standard for WBANs where ECS for WBAN is proposed.

\section{IEEE 802.15.6 WBAN Standard}

The IEEE 802.15.6 standard defines one MAC and supports three PHYs: NarrowBand (NB), human body communications (HBC), and Ultra-Wide Band (UWB) layers [30,31]. Figure 3 illustrates the IEEE 802.15.6 standard MAC and the physical layers model. The choice of physical layer in WBAN depends on the application and its requirement. NB-PHY supports various frequency bands, and WBAN devices must be able to support transmission on at least one of those frequency bands. The details of transmission parameters for NB-PHY, including frequency band, modulation, symbol rate, and ECC found at [30]. HBC-PHY uses Electric Field Communication (EFC) technology and the band of operation centered at $21 \mathrm{MHz}$. Transmission at this layer was performed using digital circuits and electrode. That makes the receiver movement easy and reduces power consumption at the receiver.

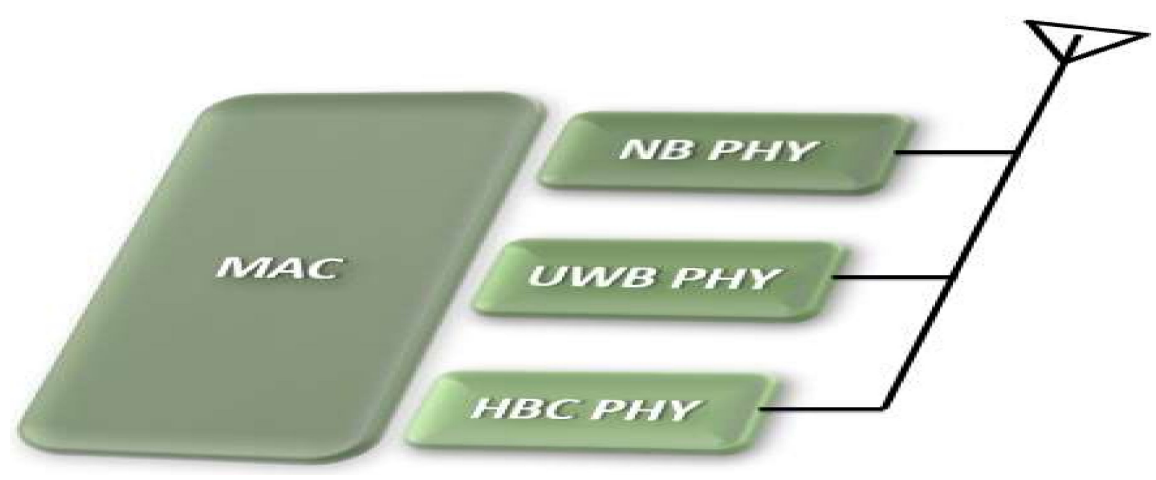

Figure 3. IEEE 802.15.6 Media Access (MAC) and Physical (PHY) layer model.

UWB-PHY is designed for high performance, low complexity, and low power consumption as compared with NB-PHY and HBC-PHY. This physical layer offers Impulse Radio UWB (IR-UWB) and wideband Frequency Modulation UWB (FM-UWB) technologies. In addition, UWB-PHY defines two 
modes of operations, called default mode and high QoS mode. The default mode used in medical and non-medical applications, where the particular QoS is not well-defined. The high QoS mode is used for high-priority medical data or network control management data. Table 1 illustrates the user priority for different types of data [30]. The standard have not been tailored to ensure the reliability for each user priority and the handling of multiple user priority.

Table 1. Quality of Service (QoS) for IEEE802.15.6 [30].

\begin{tabular}{|c|c|c|c|}
\hline Priority & User Priority & Traffic Designation & Frame Type \\
\hline \multirow{7}{*}{ Lowest } & 0 & Background (BK) & Data \\
\hline & 1 & Best Effort (BE) & Data \\
\hline & 2 & Excellent Effort (EE) & Data \\
\hline & 3 & Video $(\mathrm{VI})$ & Data \\
\hline & 4 & Voice $(\mathrm{VO})$ & Data \\
\hline & 5 & Medical or network control & Data or management \\
\hline & 6 & $\begin{array}{l}\text { High-priority medical data or } \\
\text { network control }\end{array}$ & Data or management \\
\hline Highest & 7 & $\begin{array}{c}\text { Emergency or medical implant } \\
\text { event report }\end{array}$ & Data \\
\hline
\end{tabular}

In IEEE 802.15.6, the BCH $[17,18]$ code is adopted as an ECC. In NB-PHY, BCH $(63,51)$, and its shortened version $\mathrm{BCH}(31,19)$ is used for payload and header, respectively. The modulation for narrowband PHY can be varied among Differential Binary Phase Shift Keying (DBPSK), Differential Quadrature Phase Shift Keying (DQPSK), Differential eight-Phase Shift Keying (D8PSK), and Gaussian Minimum Shift keying (GMSK) to support various data rate for payload and frequency bands.

In UW-PHY, BCH $(63,51)$ is adopted as a FEC for the default mode, whereas shortened BCH $(126$, 63) with the HARQ mechanism is adopted as ECSs for high QoS mode. Table 2 illustrates the summary of modulation, FEC, and use of HARQ in default and high QoS modes in UWB-PHY. For the detailed operation and features of MAC layer and all PHY layers refer to the standard [30]. The error correction on WBAN communication can be performed at either PHY or MAC or both layers. In WBAN, there are different types of nodes and those nodes may be inside, outside, or on the human body. Therefore, the different types of channel models are introduced to the model channel between nodes and networks to represent the overall WBAN.

Table 2. Modulation, Error-Correcting Codes (ECC), and use of Hybrid Automatic Repeat Request (HARQ) for each mode in Ultra-Wide Band-Physical (UWB-PHY).

\begin{tabular}{|c|c|c|}
\hline $\begin{array}{l}\text { Modulation/Error } \\
\text { Correction Technique }\end{array}$ & Default Mode & High QoS Mode \\
\hline Modulation & $\begin{array}{c}\text { Mandatory: on-off keying } \\
\text { Option: DPSK }\end{array}$ & $\begin{array}{l}\text { Mandatory: DPSK } \\
\text { Option: on-off keying }\end{array}$ \\
\hline FEC & $(63,51) \mathrm{BCH}$ code & $(126,63)$ Shortened BCH code \\
\hline HARQ & Not use & Use \\
\hline
\end{tabular}

\section{Channel and Network Models}

In WBAN, there are three types of nodes: implant node (placed inside human body or below the skin surface), body surface node (on the skin surface or at most two centimeters away from the body surface), and external node (not in contact with human body i.e., between a few centimeters to up to five meters away from the body surface). Therefore, the communication between these nodes creates different types of communication links, such as communication through body, Line of Sight (LOS), and Non Line of Sight (NLOS) scenarios. The possible communication links for WBAN is shown in Figure 4 [32] and there are various types of channel model (CM) to present these communication links. The channel models $\mathrm{CM}_{1}$ and $\mathrm{CM}_{2}$ characterize the communication channel 
between implant-to-implant node and implant node to body surface or external node, respectively. $\mathrm{CM}_{3}$ channel model characterizes the communication link between body surface node to body surface node, including LOS and NLOS scenarios. The $\mathrm{CM}_{4}$ channel model describes the communication link between body surface node to external node, including LOS and NLOS scenarios. The possible scenarios with description, used frequency band, and channel model are listed in Table 3 [32]. The scenarios are determined by the types of nodes involved in the communicating link and whether or not there is LOS between the nodes. Afterwards, the scenarios are grouped into classes as CMs.

All nodes are in and around the human body with a maximum range of five meters and star topology is adapted as a network topology in WBAN [30]. In this topology, one of the communication node acts as a hub and the rest of the communication nodes are used as nodes. The hubs are responsible for medium access and power management. Figure 5 illustrates that one-hop star WBAN topology for single WBAN. In a one-hop star WBAN, frame exchanges occur only between nodes and the hub of the WBAN. In practical settings, there may be more than one WBAN that may cause interference to each other. One-hop star WBAN extended to two-hop extended star WBAN, except in the Medical Implant Communication Service (MICS) band i.e., $402-405 \mathrm{MHz}$, as illustrated in Figure 6. In the extended two-hop extended network, the hub and node exchange frames optionally via a relay-capable node [30].

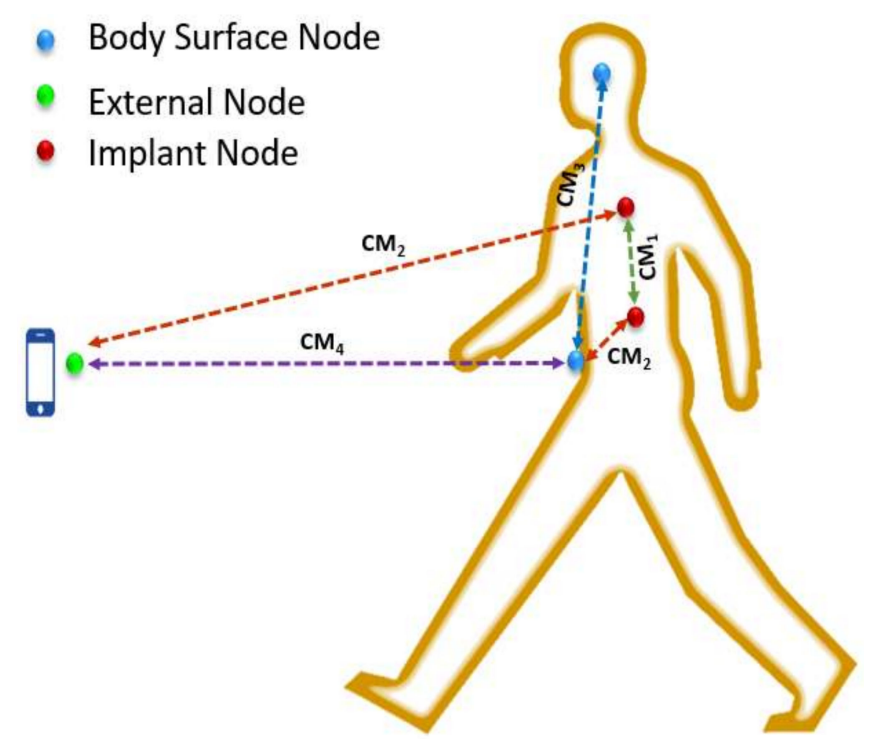

Figure 4. Possible communication links for WBAN.

Table 3. List of scenarios and their descriptions of channel models for WBAN [32].

\begin{tabular}{|c|c|c|c|}
\hline Scenario & Description & Frequency Band & Channel Model \\
\hline S1 & Implant to Implant & $402-405 \mathrm{MHz}$ & $\mathrm{CM}_{1}$ \\
\hline S2 & Implant to Body Surface & $402-405 \mathrm{MHz}$ & $\mathrm{CM}_{2}$ \\
\hline $\mathrm{S} 3$ & Implant to External & $402-405 \mathrm{MHz}$ & $\mathrm{CM}_{2}$ \\
\hline $\mathrm{S} 4$ & Body Surface to Body Surface (LOS) & $\begin{array}{c}13.5,50,400,600,900 \mathrm{MHz} \\
2.4,3.1-10.6 \mathrm{GHz}\end{array}$ & $\mathrm{CM}_{3}$ \\
\hline S5 & Body Surface to Body Surface (NLOS) & $\begin{array}{c}13.5,50,400,600,900 \mathrm{MHz} \\
2.4,3.1-10.6 \mathrm{GHz}\end{array}$ & $\mathrm{CM}_{3}$ \\
\hline S6 & Body Surface to External (LOS) & $\begin{array}{c}900 \mathrm{MHz} \\
2.4,3.1-10.6 \mathrm{GHz}\end{array}$ & $\mathrm{CM}_{4}$ \\
\hline S7 & Body Surface to External (NLOS) & $\begin{array}{c}900 \mathrm{MHz} \\
2.4,3.1-10.6 \mathrm{GHz}\end{array}$ & $\mathrm{CM}_{4}$ \\
\hline
\end{tabular}




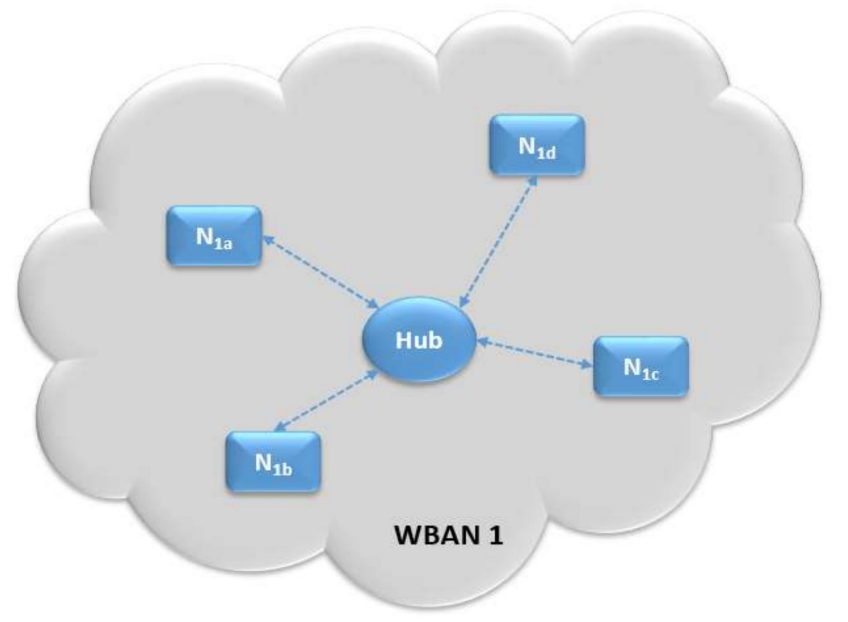

Figure 5. One hop star WBAN topology.

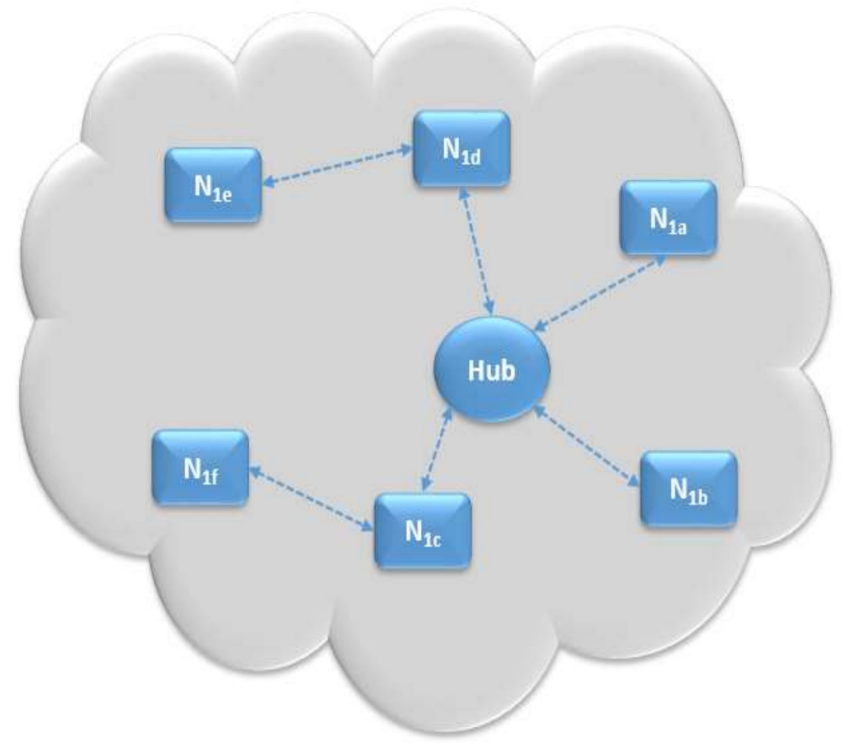

Figure 6. Two-hop extended star network WBAN topology.

\section{Review on ECS Methods on WBAN}

The IEEE 802.15.6 standard allows for performing error correction on WBAN communication at the bottom two layers of OSI. After reviewing several error correction approaches that are offered in WBAN in the literature, it is found that the classification of ECS is based on error correction techniques: FEC, HARQ, including HARQ-I and HARQ-II, and modulation order. ECS methods that are introduced in the literature are classified based on use of OSI layer that is displayed on Table 4.

Table 4. ECS classification based on Open Systems Interconnection (OSI) layers.

\begin{tabular}{clc}
\hline OSI Layer & & ECS Methods \\
\hline PHY & FEC and modulation & $\begin{array}{c}\text { Cross layer: ARQ, HARQ, FEC/ARQ/HARQ } \\
\text { combined with access control mechanism }\end{array}$ \\
\hline MAC & FEC applied to MAC & conn
\end{tabular}

\subsection{Forward Error Correction (FEC)}

As per the WBAN standard, error correction on WBAN communication is performed at bottom two layers of OSI. From the literature review, we also observed that FEC could be applied to the bottom 
two layers: PHY or MAC. In [33-36], FEC is applied on PHY layer only, whereas in [37] the same is applied at the MAC layer only.

In [38], the authors presented the experimental result of measured RSS around a human body, keeping two antennas attached to the body at $444.5 \mathrm{MHz}$ and $2.45 \mathrm{GHz}$ in an anechoic chamber i.e., with very limited interference. Subsequently, the measured RSS value is used to calculate BER and PER using Aglient' Advanced Design System (ADS) Ptolemy solution. In [33,34], the authors extended their work of [38] and presented the measured RSS result around a human body keeping two antennas attached to the body at $444.5 \mathrm{MHz}[33]$ and $2.45 \mathrm{GHz}[33,34]$. Next, the measured RSS value in the office room used for fading duration, and consequently the packer erasure ratio corresponding to various data rate for both frequency bands when considering various receiver's sensitivity threshold values [33]. Following, the paper introduced the Luby Transform (LT) code as rateless code as an erasure correction code and presented a theoretical analysis of retransmission probability for various data rates and packet sizes under an erasure channel. The packer erasure ration that was calculated from the measured RSS measurement value around human body and the design criteria that were developed for the rateless code are isolated work. The result presented is a standalone performance of the rateless code without considering real WBAN setting.

In [34], the measured RSS values used to calculate PER versus various data rate. Subsequently, the authors proposed LT codes as an error mitigation technique to achieve high reliability in WBAN during the shadowing effect. The paper also presented theoretical throughput of LT and RS codes versus the number of transmitting packets. The authors claim that LT code outperforms the RS code in WBAN when the probability error rate of the channel is high due to the shadowing effect. However, RS codes perform better than LT codes in other conditions. The PER calculation from the measured RSS measurement value around the human body and the throughput performance result of the two codes are isolated work. The presented throughput results of those codes is a standalone performance of these codes without considering the real WBAN setting.

In [35], the authors proposed an adaptive ECC to select ECC according to channel conditions and different type of transmission data, including medical and non-medical data. The requirement of different QoS for the different types of transmission data is considered. The authors proposed convolutional code $(2,1)$ for AWGN channel and concatenated RS $(63,51)$-convolutional code $(2,1)$ codes for Rician and Rayleigh channels for medical data to meet the desired QoS, respectively. Whereas, the RS $(63,51)$ code for the AWGN channel and concatenated convolutional code $(2,1)$ codes for Rician and Rayleigh channels are proposed for non-medical data to meet the desired QoS. For medical data, the paper recommended using the convolutional code $(2,1)$ against RS $(63,51)$ code in the AWGN channel, despite that both codes are achieving the required QoS and RS $(63,51)$ having a higher coding rate, but the same reason is given for code selection in other scenarios. Hence, their method of code selection is not consistent. The paper proposed channel estimation using a pilot signal, but it does not provide details, including its impact on WBAN setting.

The BER results of the BCH code and that of the proposed codes over different channels are standalone performances of those codes without considering the WBAN setting. The paper did not outline what type of decoding algorithms is used during the BER performance of convolutional code. As we discussed in Section 2.2, the performance changes significantly when decoding algorithm changes from hard-decision to soft-decision during decoding. The code rate of the proposed code is significantly lower when compared with the standard $\mathrm{BCH}$ code. The performance of ECC improved when the code rate is reduced. The code rate of 0.5 is convolutional code $(2,1)$ for AWGN channel and the code rate is 0.4 for concatenated RS $(63,51)$-convolutional code $(2,1)$ codes for Rician and Rayleigh channels for medical data to meet the desired QoS, which is significantly lower than the code rate of 0.8 for the $\mathrm{BCH}$ code. In general, the error control capability of the ECC is enhanced when the code rate is lower for any code. Therefore, to make fair comparison, the paper should have a presented BCH code with a similar rate. Therefore, the result is not a fair comparison when comparing the performance of code with different rates in the same setting. 
In [36], the authors simulate the performance evaluation LT codes in WBAN and compared the result with $\mathrm{BCH}$ codes with a comparable rate. The author claimed that the $\mathrm{BCH}$ code outperformed LT codes for a higher code rate, but the LT code outperformed the $\mathrm{BCH}$ code for lower code rate in several WBAN settings. The claim was developed from BER simulation results that considered SNR values from 0 to $40 \mathrm{~dB}$. However, this is very unrealistic when WBAN is energy-constrained. Second, the BER simulation result of the coded (various rates) and uncoded system presented against SNR, which is not a fair comparison, as we explained in Section 2.2. The paper also presented PAR for both codes against hop distance ranging from 10 to $40 \mathrm{~cm}$ only. The range of hoping distance is very low when compared with the average size of the human body. However, the PAR reached almost zero when the hoping distance reached the upper value of hoping distance for all codes. The result indicated that the proposed code could provide a PAR of more than 0.9 in WBAN communication only around $10 \mathrm{~cm}$ hoping distance that is not suitable for average human body size.

In [37], the authors conducted an initial investigation on the application of RS code on MAC layer data using real data of MIT-BIH arrhythmia databases. However, most of the paper content is a review of the IEEE 802.15.6 standard. The BER performance of shortened RS codes with varying code rate from 0.74 to 0.98 over the AWGN channel and fading channel is presented. However, the type of fading is not specified. The presented result and conclusion that are drawn in the paper are very generically related with coding principles but are not specific to the WBAN setting.

In [39], the authors presented the Raptor code as an ECC for WBAN and compared its performance (in term of energy consumption versus distance) with $\mathrm{BCH}$. The paper claimed that the energy efficiency of the proposed code is better than $\mathrm{BCH}$ for different types of fading channels, including Nakagami, Rayleigh, and Rician, when the distance between nodes is higher than $10 \mathrm{~m}$. However, WBAN is network around a human body and the range of the distance between nodes in the network is limited to less than five meters in practical settings. In [40], the authors extended their work [39], where the result is compared with two more techniques: LT code and ARQ. These presented results are standalone performance without considering the WBAN setting.

The authors introduced a static XOR (logical operation) based architecture with three relays selection for WBANs in [41]. The BER results that are presented in [41] are against SNR instead of $E_{b} / N_{0}$. Hence, the results do not take the code rate in to consideration i.e., do not consider the overhead that is caused by ECS. The decoding algorithm is performed by the BP algorithm, the cost that is associated with BP decoding is not presented, and how this decoding algorithm is suitable for energy-constrained WBAN scenarios is not clear. Furthermore, it is also not clear what type of networks are considered during the simulation.

The paper proposed a low complexity version of bit flipping algorithm, called modified implementation efficient reliability ration WBF for LDPC code for implant to implant communication channel in WBAN [42]. The authors presented a decoding process in the proposed algorithm. Subsequently, the performance of the proposed decoder is compared with various versions of LDPC decoders, including the min-sum, WBF, and various versions of WBF in terms of BER and the required number of iterations required to converge against $E_{b} / N_{0}$. The performance of the proposed code is better than other versions of WBF decoding algorithms in both factors. Additionally, the paper presented required the decoding time against $\mathrm{E}_{\mathrm{b}} / \mathrm{N}_{0}$ for different rates LDPC codes with several version of WBF, min-sum, and the proposed algorithm. The decoding time for the proposed code lays medium in range when compared with others. The work that is presented in [42] is limited to implant to implant node communication channel only and there is only comparison with different versions of LDPC decoders. However, there is a lack comparison with other types of codes that has similar complexity and is considered for WBAN.

The authors introduce a framework to connect two WBANs using UMTS in [43] that supports various rates according to QoS requirements. The authors combined convolutional code in WBAN with UMTS codes of cellular in the proposed framework. In the proposed framework, the medical data uses the adaptive convolutional code and UMTS code, whereas non-medical data only use the UMTS 
code. The proposed code rates for convolutional code are half, one-third, and a quarter for higher QoS, medium QoS, and lower QoS medical data, respectively. The soft-decision decoder Viterbi algorithm is used in the decoding operation in the proposed system. However, the complexity that is introduced by the use of soft-decision decoder is not analyzed. The theoretical upper bound bit error probability for up and downlink channels when considering AWGN and Rayleigh channels are presented without comparing with other results. The result is a standalone result without taking any consideration of other WBAN and interferences.

A summary of FECs based ECS is presented on Table 5. From the literature review, we observed that all of the proposed ECS were considering only a single WBAN without considering interference from other WBAN that is not realistic or practical setting. More research has to be conducted when considering multiple WBANs or considering the interference from other existing WBANs.

Table 5. A review of forward error correction (FEC) based ECS.

\begin{tabular}{|c|c|c|c|}
\hline Ref. (Yr) & Proposed FEC & WBAN/Standard/Layer/Data/Channel & Results \\
\hline [33] (010) & LT & $\begin{array}{l}\text { One hop star network and single WBAN/IEEE } \\
\text { 802.15.6 Task Group/PHY/no discussion/erasure }\end{array}$ & $\begin{array}{l}\text { Packet erasure ratio, } \\
\text { retransmission } \\
\text { probability }\end{array}$ \\
\hline [34] (012) & LT & $\begin{array}{l}\text { One hop star network and single WBAN/IEEE } \\
\text { 802.15.6 Task Group/PHY/no discussion/erasure }\end{array}$ & $\begin{array}{l}\text { PER, retransmission } \\
\text { probability and } \\
\text { throughput LT and } \\
\text { RS codes }\end{array}$ \\
\hline [35] (016) & $\begin{array}{l}\text { RS, convolutional code } \\
\text { and Concatenated code }\end{array}$ & $\begin{array}{l}\text { One hop star network and single WBAN/IEEE } \\
\text { 802.15.6/PHY/medical and non-medical/AWGN, } \\
\text { Rayleigh and Rician fading }\end{array}$ & BER and coding gain \\
\hline [36] (015) & LT & $\begin{array}{c}\text { One hop star network and single WBAN/IEEE } \\
\text { 802.15.6/PHY/no discussion/channel } \\
\text { adapted from }[41,44,45]\end{array}$ & $\begin{array}{l}\text { BER, coding gain and } \\
\text { packet acceptance rate }\end{array}$ \\
\hline [37] (013) & RS & $\begin{array}{l}\text { One hop star network and single WBAN/IEEE } \\
\text { 802.15.6/MAC/no discussion/AWGN and fading }\end{array}$ & BER \\
\hline$[39,40](017,018)$ & Raptor code & $\begin{array}{c}\text { One hop star network and single WBAN/IEEE } \\
\text { 802.15.6/PHY/no discussion/Nakagami, Rayleigh } \\
\text { and Rician }\end{array}$ & Energy consumption \\
\hline [41] (016) & Linear block code & $\begin{array}{c}\text { Two hop star network and single WBAN/no } \\
\text { discussion/PHY/no discussion/Rayleigh and } \\
\text { Rician }\end{array}$ & BER \\
\hline [42] & $\begin{array}{l}\text { LDPC with modified } \\
\text { WBF }\end{array}$ & $\begin{array}{l}\text { One hop star network and single WBAN/IEEE } \\
\text { 802.15.6/PHY/no discussion/Implant to implant }\end{array}$ & BER, and no of iterations \\
\hline [43] (018) & $\begin{array}{l}\text { Adaptive } \\
\text { convolutional code }\end{array}$ & $\begin{array}{c}\text { One hop star network and single WBAN/IEEE } \\
\text { 802.15.6/PHY/medical and non-medical/AWGN } \\
\text { and Rayleigh }\end{array}$ & $\begin{array}{l}\text { Theoretical bit } \\
\text { error probability }\end{array}$ \\
\hline
\end{tabular}

\subsection{HARQ}

As we discussed in Introduction, WBAN standard permits perform error correction using FEC or HARQ, depending on type of PHY layers. In UWB, error correction can used both techniques, depending upon the mode of communications (refer to Table 2). From the literature review, it is observed that the researchers have applied both HARQ-I and HARQ-II error correction techniques in WBAN communications. In both of these approaches, bottom two layers of OSI are involved.

\subsubsection{HARQ-I}

In [46], the authors proposed adaptive HARQ-I for the wireless control of medical equipment to get high reliability communications between controller and sensors node. In the proposed technique, HARQ-I combines convolutional code with ARQ. The paper assumed that the wireless control system works as a rotated inverted pendulum. In the proposed system, the code rate adaption (three possible rates) of the convolutional code is performed using puncturing according to the importance of quantity of state of the control system according. The control quality is improved by the method of limitation of retransmission. The paper presented the comparison result of MAE and the rate of increase of 
frequency band versus SNR over AWGN channel for HARQ-I and showed the proposed adaptive HARQ-I. The proposed system performs slightly better than HARQ-I. The presented results of the proposed method are standalone performance without considering the WBAN setting. Table 6 shows the summary of HARQ-I based ECS.

Table 6. A review of HARQ-I based ECS.

\begin{tabular}{cccc}
\hline Ref. (Yr) & Proposed Technique & WBAN/Standard/Layer/Data/Channel & Results \\
\hline [46] (010) & $\begin{array}{c}\text { Adaptive convolutional } \\
\text { code with ARQ }\end{array}$ & $\begin{array}{c}\text { One hop star network and single } \\
\text { WBAN/IEEE 802.15.6/Cross layer/no } \\
\text { discussion/AWGN }\end{array}$ & $\begin{array}{c}\text { MAE and rate of increase } \\
\text { of frequency band }\end{array}$ \\
\hline [47] (016) & BCH with adaptive ARQ & $\begin{array}{c}\text { One hop star network and single } \\
\text { WBAN/IEEE 802.15.6/Cross } \\
\text { layer/NA/AWGN }\end{array}$ & $\begin{array}{c}\text { Energy consumption and } \\
\text { delivery ratio }\end{array}$ \\
\hline
\end{tabular}

In [47], the authors introduced the error control strategy for WBAN, while ultrasonic waves were used as a communication medium. The paper considers three types of strategies: ARQ only, HARQ-I (called A\&F that combines ARQ with fixed maximum retransmission and $\mathrm{BCH}$ ), and adaptive HARQ-I (called UECS that combines ARQ with adaptive retransmission and $\mathrm{BCH}$ ). This work presented the energy consumption and delivery ratio of ARQ only, A\&F, and UECS against SNR. Consequently, the results do not take the code rate of the adaptive coding into consideration i.e., do not consider the overhead that is caused by ECS. Therefore, the comparison that is made in [47] is not fair and reasonable. Additionally, the authors did not consider energy consumption due to encoding and decoding that is the key drawbacks of this paper. Furthermore, the adaption is performed using SNR estimation and the cost that is associated with channel estimation is not considered during energy consumption estimation. The presented results are standalone results without considering the practical WBAN setting.

\subsubsection{HARQ-II}

We present a review of HARQ-II based ECS in Table 7. In [48], the authors introduced adaptive HARQ-II for WBAN based on UWB-PHY. Figure 7 illustrates the concept that is presented in paper [48]. The paper considered the different types (medical and non-medical data) with different QoS requirements data transmission. The coding, modulation, and demodulation scheme is the same for both types of data transmission. The reliability of medical data is enhanced by the use of selective ARQ and by transmitting the parity bits and data bits alternatively during retransmission when NACK was received from the receiver. However, ARQ is not applied to non-medical data transmission. The author claimed that the proposed scheme is suitable for the IEEE 802.15.6 CM3 (on-body to on-body devices) scenario and the same is considered during the simulation. However, CM3 incurs different types of channels (refer to Table 3), such as LOS and NLOS. The paper did not outline what type of specific channel has been used during the simulation.

In [48], BCH $(63,51)$ is used as ECC and binary pulse position modulation (2PPM) as modulation in HARQ-II. In the MAC layer, the paper considers medium access control methods of slotted ALOHA and polling during the simulation. The performance of the proposed system measured in terms of the probability of accepting error frame rate and buffer usages versus SNR. The result indicated that the use of polling improves the performance during the presence of multiple users when compared with slotted ALOHA. However, the simulation result of throughput efficiency versus maximum number of transmission showed that the use of slotted ALOHA perform better than polling in the high SNR region. The presented results of the proposed method are standalone performance without considering WBAN network settings. 
Table 7. A review of HARQ-II based ECS.

\begin{tabular}{|c|c|c|c|}
\hline Ref. (Yr) & Proposed Technique & WBAN/Standard/Layer/Data/Channel & Results \\
\hline [48] (010) & $\begin{array}{l}\text { BCH code with } \\
\text { selective ARQ }\end{array}$ & $\begin{array}{l}\text { One hop star network and single } \\
\text { WBAN/IEEE 802.15.6/Cross layer/medical } \\
\text { and non-medical/CM3 (on-body to } \\
\text { on-body) }\end{array}$ & $\begin{array}{l}\text { Accepting frame error } \\
\text { rate, buffer usage and } \\
\text { throughput efficiency }\end{array}$ \\
\hline [49] (013) & $\begin{array}{l}\text { Reed-Muller code with } \\
\text { Weldon' s ARQ }\end{array}$ & $\begin{array}{c}\text { One hop star network and single } \\
\text { WBAN/IEEE } 802.15 .6 / \text { Cross layer/medical } \\
\text { and non-medical/CM3 (on-body to } \\
\text { on-body) }\end{array}$ & $\begin{array}{l}\text { Residual BER } \\
\text { and throughput }\end{array}$ \\
\hline [50] (013) & $\begin{array}{l}\text { Convolutional code with } \\
\text { Weldon's ARQ }\end{array}$ & $\begin{array}{l}\text { One hop star network and single } \\
\text { WBAN/IEEE 802.15.6/Cross layer/medical } \\
\text { and non-medical/AWGN }\end{array}$ & $\begin{array}{l}\text { Residual BER } \\
\text { and throughput }\end{array}$ \\
\hline$[51,52](015)$ & $\begin{array}{l}\text { Convolutional code with } \\
\text { Weldon's ARQ }\end{array}$ & $\begin{array}{c}\text { One hop star network with multiple } \\
\text { WBANs/IEEE 802.15.6/Cross } \\
\text { layer/medical and non-medical/AWGN }\end{array}$ & $\begin{array}{l}\text { Residual BER } \\
\text { and throughput }\end{array}$ \\
\hline [52] (015) & $\begin{array}{l}\text { Convolutional code with } \\
\text { Weldon's ARQ }\end{array}$ & $\begin{array}{l}\text { One hop star network and single } \\
\text { WBAN/IEEE 802.15.6/Cross layer/medical } \\
\text { and non-medical/AWGN }\end{array}$ & $\begin{array}{l}\text { Residual BER, } \\
\text { throughput and } \\
\text { energy efficiency }\end{array}$ \\
\hline [53] (016) & $\begin{array}{l}\text { Convolutional code with } \\
\text { Weldon's ARQ }\end{array}$ & $\begin{array}{c}\text { Multihop star network and single } \\
\text { WBAN/IEEE 802.15.6/Cross layer/medical } \\
\text { and non-medical/AWGN }\end{array}$ & $\begin{array}{l}\text { PER, energy efficiency } \\
\text { and average number of } \\
\text { transmission }\end{array}$ \\
\hline [54] (017) & $\begin{array}{l}\text { Convolutional code with } \\
\text { Weldon's ARQ }\end{array}$ & $\begin{array}{c}\text { One hop star network and single } \\
\text { WBAN/IEEE } 802.15 .6 / \text { Cross layer with } \\
\text { different access techniques/medical and } \\
\text { non-medical/AWGN }\end{array}$ & $\begin{array}{l}\text { Average number of } \\
\text { transmission and } \\
\text { probability of } \\
\text { unsuccessful } \\
\text { transmission }\end{array}$ \\
\hline [55] (016) & $\begin{array}{l}\text { Convolutional code with } \\
\text { Weldon's ARQ }\end{array}$ & $\begin{array}{l}\text { One hop star network and single } \\
\text { WBAN/IEEE 802.15.6/Cross layer/medical } \\
\text { and non-medical/AWGN, Rayleigh }\end{array}$ & $\begin{array}{l}\text { Bit error probability, PER, } \\
\text { throughput, } \\
\text { residual BER, }\end{array}$ \\
\hline
\end{tabular}

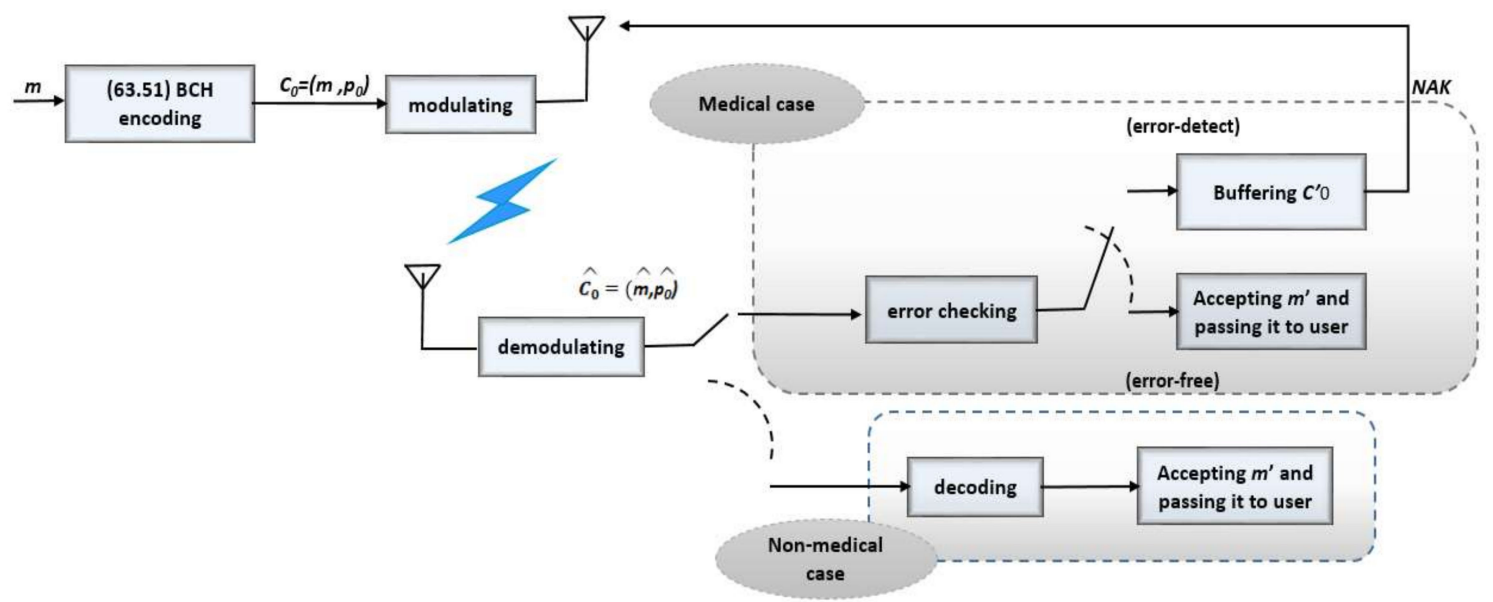

Figure 7. Hybrid automatic repeat request-II (ARQ-II) [48].

In [50,56], the authors proposed the combination of decomposable codes (based on convolutional code in [50] and Reed-Muller codes in [49]) and Weldon's ARQ to adjust the balance between error correcting capability and the number of retransmission according to required QoS, depending on data types. The concept of ECS that is used in these papers is HARQ-II that is similar to [48]. However, these papers uses the Reed-Muller code [49] and convolutional code [50] instead of BCH code, Weldon' ARQ instead of selective ARQ, and only considers medical data with different user priority levels. In [50], the paper outlines the process of multiplexing of various data types i.e., data with different user priority, as illustrated in Figure 8. In both papers, the operation of Weldon's ARQ and respective decomposable ECCs are shown. In [50], the comparison results is presented in terms of residual BER 
and throughput performance versus $E_{b} / N_{0}$ over AWGN channel for the proposed and conventional method i.e., use of selective-repeat ARQ. The paper claimed that the proposed scheme is having better performance than the conventional method. The code rate is adaptive in the order of $7 / 8$, $5 / 6,3 / 4,1 / 2$. In [49], the results are presented in terms of residual BER and throughput performance versus $E_{b} / N_{0}$ for the proposed scheme. However, no comparison with any other results is presented. The paper [49] claimed that IEEE 802.15.6 CM3 is used during the simulation, but CM3 covers different types of channels (refer to Table 3), such as LOS and NLOS. The paper did not outline what type of specific channel has been used during the simulation. The residual BER and throughput performance versus $E_{b} / N_{0}$ for the proposed scheme, but with no comparison with any other result is presented. The adaptive code rate of convolutional code [50] and Reed-Muller code [49] are considered during the simulation, but how $E_{b} / N_{0}$ is evaluated during the simulation is not clear in both papers. Furthermore, the papers did not outline the types of decoding algorithm used in during the channel decoding that is also key factor for energy-constrained node in WBAN.

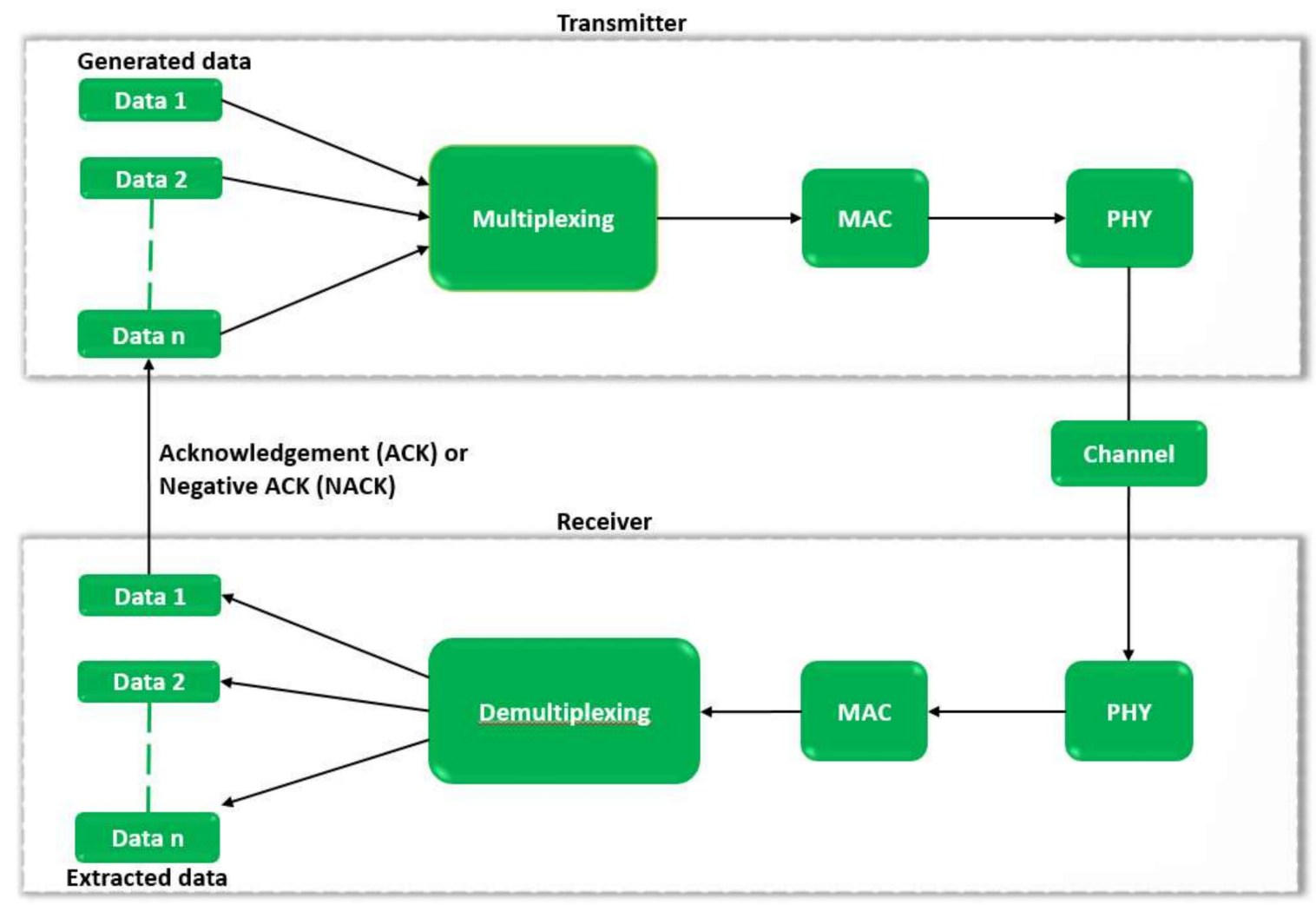

Figure 8. Block diagram for multiplexing $[50,56]$.

The concepts introduced at [50] are extended in [51,52] when considering interference from the multiple WBAN environment. Two cases are considered in multiple WBAN environment: average and worst case. In average case, interfering WBANs are uniformly distributed within three meters from the objective WBAN, whereas interfering WBANs are closely placed to the objective WBAN in the worst case. In this paper, the compared results are presented in terms of residual BER and throughput performance versus $E s / N_{0}$ for the proposed and conventional methods i.e., IEEE 802.15.6 approach. In the proposed scheme, both types of data (user priority 5 and 6 ) is using the convolutional code with HARQ-II and code rate varying from $8 / 9$ to $1 / 6$, but with a different number of maximum retransmission according to user priority of data. In the standard approach, data with user priority 5 using $\mathrm{BCH}(63,51)$ with code rate 0.8 , but data with high priority uses $\mathrm{BCH}(126,63)$ with a HARQ-II i.e., code rate of 0.5 and lower. The results (residual BER and throughput) are presented in $[51,56]$ is against $E s / N_{0}$ instead of $E_{b} / N_{0}$. Consequently, the results do not take the code rate in to consideration i.e., do not consider the overhead caused by ECS. However, the same authors presented the results 
(residual BER and throughput) against $E_{b} / N_{0}$ in $[50,56]$ in similar settings. Therefore, the comparison made in $[51,56]$ is not fair and not reasonable, as we discussed in Section 2.2. In addition, the simulation settings in the paper indicated that the channel model are considered as CM3 and CM4, the detailed discussion of how those channel models are adjusted in the simulation is not presented. Furthermore, the proposed scheme uses soft-decision Viterbi decoding that has a significantly higher decoding cost when compared with standard $\mathrm{BCH}$ decoding. However, the cost associated with the decoding complexity is not considered during the result presentation and discussion.

The work presented in [52] is similar to [50], with slight variations and some additional work. The additional work performed in [52] is an energy efficiency evaluation of the proposed ECS that is based on [57]. The energy efficiency as a function of $E S / N_{0}$ is presented for the proposed ECS and standard approach for data with different priorities (user priority 5 and 6). However, the energy efficiency evaluation of the standard approach i.e., IEEE 802.15.6 approach is presented without proper evaluation in the paper. The energy efficiency is presented as a function of $E s / N_{0}$ that do not consider the overhead that is caused by the code rate of ECS. The simulation setting for the average case in $[51,56]$ and [52] is almost the same, but the residual BER of data with higher user priority has $2 \mathrm{~dB}$ coding gap between results in [51,56] and [52]. However, the paper did not discuss discrepancy in the result, even if the main author of both papers is same. In [52], the paper presented residual BER and energy efficiency versus allowed delay for different values of $E s / N_{0}$. The author concluded that the residual BER of the proposed scheme was improved for poor channel condition that is very predictable as the proposed scheme is using the complex decoding algorithm as well as lower code rate ECC when compared to the standard approach. In addition, residual BER and energy efficiency [52] are presented versus $E s / N_{0}$ and the discussion also only refers to $E s / N_{0}$ instead of $E_{b} / N_{0}$ that do not incorporate overhead that is caused by ECS. Therefore, the presented result is not a fair comparison.

The concepts that are introduced at [50] are also extended in [53] when considering multihop WBAN (refer to Figure 6). The concept of Weldon's ARQ operation is extended to two hops WBAN setting. The performance of the proposed scheme is compared with standard scheme (IEEE 802.15.6 approach) in terms of PER, energy information, and average number of transmission versus $E s / N_{0}$ while considering the DBPSK and DQPSK modulation scheme. The paper claimed that the proposed scheme has better performance than the standard approach. However, the paper uses binary and higher order modulations as well as various code rates, but the results are presented against $E s / N_{0}$ instead of $E_{b} / N_{0}$. Referring to Equations (2) and (3), the results do not reflect the code rate and higher order modulation when they are presented against $E s / N_{0}$. Therefore, the presented results do not consider code rate and higher order modulation during the result presentation. In addition, result is not considering the decoding complexity of soft decision Viterbi decoding during the result and discussion.

In [54], the authors extend their previous work [50] when considering two types of access mechanism (random and schedule) in the MAC layer. The probability of successful transmission on PHY, MAC, and cross-layer (considering PHY and MAC) is derived mathematically. In the paper, the compared results are presented in terms of average number of transmissions and probability of unsuccessful transmission versus Es $/ \mathrm{N}_{0}$ for the proposed and standard method i.e., IEEE 802.15.6 approach. The paper claimed that the proposed scheme has better performance than the standard approach. However, the result does not take code rate into account, as the result is presented against $E s / N_{0}$ instead of $E_{b} / N_{0}$. Additionally, complex decoding algorithms are used in the proposed system without considering the decoding cost. Therefore, the claim of better performance is not fair and straightforward as mentioned in the paper.

In [55], the authors extend their work $[50,56]$ in two aspects. Previous works are only based on simulation under AWGN channel. In [55], the authors presented a theoretical analysis based on bit error probability and event error probability of the proposed schemes in $[50,56]$ under AWGN and Rayleigh fading channels. The theoretical results indicated that both error probabilities for both channels as a function of code rate and $E_{b} / N_{0}$ that is exactly we discussed in Equations (2) and (3). However, the simulation results of bit error probability and throughput is presented against Es $/ \mathrm{N}_{0}$ 
that is against their theoretical findings where error probabilities for both channels are presented as a function of $E_{b} / N_{0}$. Secondly, the decomposable code used in previous work is slightly modified to perform theoretical analysis. The concern of using $E s / N_{0}$ instead of $E_{b} / N_{0}$ in the simulation result is raised again and the use of complex decoder without considering the decoding cost in $[51,56]$ also applies in this paper. Therefore, the simulation result that is presented in [55] did not consider the code rate and decoding complexity, while comparing the results with the IEEE 802.15.6 approach.

\subsection{Modulation}

Researchers used the modulation technique of different orders as the error correction procedure. In this technique, the adaption of different order of modulation according to channel condition is used as an ECS. In $[58,59]$, the authors introduced the link adaption technique in WBAN while considering the IEEE 802.15.4 standard to reduce packet loss where CSMA/CA in MAC and different modulation schemes in physical layer are combined. The link adaptation technique does not use any ECC and the WBAN considered in these papers is one-hop star topology. The link adaptation technique requires channel estimation at each node in the WBAN. The channel estimation in terms of SNR is performed while using received beacon power and signal to interference ratio (SIR) is evaluated while using failure probability during packet transmission. The link adaptation i.e., selection of modulation scheme (from node to coordinator) is performed using SNR only in [60] and using SNR and SIR both in [58]. The data rate adapted according to SNR only in [60] and SNR and SIR both in [58]. The important concern is the issue of channel estimation in terms of SNR and SIR in WBAN nodes. The papers discussed how to perform channel estimation at the node, but the cost that is associated with channel estimation at the computationally and energy-constrained node is not considered during the study.

We present a summary of FECs that are based on modulation in Table 8. From the literature review, we found that modulation order update is happening according to link conditions. The estimation of link condition and SIR requires a good channel estimation technique that is an energetically and computationally complex process. However, there is a lack of research on how much cost is associated with estimation and how it affects the overall cost.

Table 8. A review of modulation based ECS.

\begin{tabular}{cccc}
\hline Ref. (Yr) & Proposed Technique & WBAN/Standard/Layer/Data/Channel & Results \\
\hline \multirow{3}{*}[58]{$(010)$} & $\begin{array}{c}\text { Modulation order } \\
\text { (O-QPSK, 2,4,8-DPSK) } \\
\text { according to link }\end{array}$ & $\begin{array}{c}\text { One hop star network (considering SNR } \\
\text { and SIR)/IEEE 802.15.4/PHY/no } \\
\text { discussion/AWGN }\end{array}$ & PER \\
\hline \multirow{3}{*}{ [60] (012) } & Modulation order & One hop star network (considering & \\
& $\begin{array}{c}\text { (O-QPSK, 2,4,8,16-DPSK) } \\
\text { according to link }\end{array}$ & $\begin{array}{c}\text { SNR)/IEEE 802.15.4/PHY/no } \\
\text { discussion/AWGN }\end{array}$ & PER \\
\hline
\end{tabular}

We observed from the literature review that the researchers utilize standard techniques of error corrections to enhance reliability without considering the constraints that are faced in WBAN. Some researchers used ECS with complex decoder instead of ECS with a simple decoder. However, the decoding cost that is introduced by the complex decoder is not taken into consideration during the result. Another case to consider is that the researcher uses lower rate ECC, Which enhanced the reliability when compared with higher rate ECC, but it does not consider the code rate during the result and discussion.

In WBAN, the communications scenarios are different significantly from other communications systems in many factors. Firstly, there are different types of nodes (implant node, body surface node, and external node) and computational and energy constraints that are faced by these nodes are different, but those constraints are not exploited on the proposed ECS in the literature. In addition, there are a variety of channels types and scenarios (refer to Table 3) that exist in WBAN. In the following section, we discuss the research challenges and opportunities on ECS design for WBAN. The ECS for 
WBAN should consider computational complexity and energy constraints of nodes, different types of possible channels of WBAN, and different types of QoS requirements.

\section{Future Research Challenges and Opportunities}

In our view, the ECS should be dynamic, so that it should able to handle a variety of computational complexity, energy-constrained nature of nodes, and different types of communication channels exist in WBAN. The future ECS should exploit those node constraints and the possible communication channels during ECS design and implementation. There are three types of nodes (refer to Figure 4) and computational complexity and energy-constraints of node depends on the type of node. Implant nodes are battery operated, and thus there are strict computational limitations and energy-constrained requirements at implant nodes. However, body surface (wearable devices) and external (mobile or medical devices) nodes are using a rechargeable battery or power supplied at these nodes. Therefore, there is a fewer computational and energy-constraints requirement at these nodes when compared to implant nodes. However, the literature review in Section 5 indicates that previous works were lacking in using these factors during the design of ECS. Therefore, the ECS design and implementation for WBAN should consider those computational and energy-constrained at nodes during the design.

The suitability of ECS for any communication system depends on the type of communication channel that exist on the communication system. In WBAN, several types of communication channels exist and variation of path loss varies significantly [32,61]. Therefore, a dynamic ECS should be considered for WBAN to support a variety of channels and nodes that exist in WBAN. The dynamic ECS should able to use multiple ECS techniques in WBAN to handle varieties of QoS requirements, latency requirements, and several type of nodes and channels. Error correction codes and techniques should vary according to scenarios and channel models (refer to Table 3).

In our view, the following research areas needs to be explored to design efficient ECS design for WBAN:

- To support variety of nodes, channels, and QoS requirements in WBAN, are dynamic ECS design and implementation based on the software-defined radio suitable for WBAN as introduced for wireless sensor network in [62-65]?

- The overall efficiency of ECS for WBAN depends on interferences from other WBANs and other existing systems, such as Wi-Fi and WLAN. Further research is expected to analyse the efficiency of WBAN in practical and real-life settings rather than isolated settings, as considered in previous research works.

- A very limited research work is conducted when considering two hop WBAN. One open area of the research is to analyse the consequence, significance, and complication on ECS design when considering two hop in WBAN.

- Does treating the forward link (for example, implant node to surface node) and reverse link (for example, surface node to implant node) differently (similar to mobile communication uplink and downlink) facilitate the innovative design of ECS of WBAN?

- Most of the proposed ECS designs are based on RF communication channels. Does the HBC technique improve or aid on design of efficient ECS for WBAN? This research area, however, significantly differs from the ECS design that we discussed in this paper.

- Most of proposed research works on ECS design for WBAN are based on RF communication channels. Is the optical communication technique, as discussed in [65], appropriate to improve or aid in design of efficient ECS for WBAN specifically implant to implant and implant to wearable device? This research area, however, significantly differs from the ECS design that we discussed in this paper.

- One of the greatest challenges in ECS design on WBAN is to verify the design and expected outcome. Therefore, the creation of simulation settings or tools that resembles the real human bodies is another challenge and would be another research area to explore. 


\section{Conclusions}

In this paper, we presented a brief review of the different types ECS available in literature that are used in the communication system in the context of WBAN. A summary of the IEEE standard, channel, and network models for WBAN is also presented, which is vital for ECS design for WBAN. Subsequently, we conducted an extensive review of different types of ECS proposed in the literature for WBAN. The review has been conducted by looking into various aspects, for instance, the types of ECS used, types of WBAN, data types considered, and types of channels considered.

Finally, we offered future research challenges and opportunities that exist for ECS design for WBAN. We anticipated that the forthcoming ECS for WBAN should be dynamic and involved multiple ECS techniques in WBAN to handle varieties of QoS requirements, latency requirements, and several type of nodes and channels.

Author Contributions: Introduction-R.K., N.I., K.A. and S.J.H; ECS-R.K. and N.I.; Standard-R.K. and K.A.; Channel and Network models-R.K. and S.J.H; Review-R.K., N.I., K.A. and S.J.H.; Future research-R.K.; Conclusion-R.K. and N.I.

Funding: This research received no external funding.

Conflicts of Interest: The authors declare no conflict of interest.

\section{Appendix A}

Table A1. A list of acronyms.

\begin{tabular}{cccc}
\hline Acronyms & Definitions & Acronyms & Definitions \\
\hline ACK & Acknowledgement & MAC & Media Access \\
ADS & Advanced Design System & MAE & Mean Absolute Error \\
ARQ & Automatic Repeat Request & MAP & Maximum a Posteriori \\
AWGN & Additive White Gaussian Noise & MICS & Medical Implant \\
2PPM & Binary Pulse Position Modulation & ML & Maximum Likelihood \\
BCH & Bose-Chaudhuri-Hocquenghem & NACK & Negative Acknowledgement \\
BER & Bit Error Rate & NB & Narrow Band \\
BMP & Binary Message Passing & NLOS & Non Line Of Sight \\
BP & Belief Propagation & O-QPSK & Offset Quadrature Phase Shift Keying \\
CM & Channel Model & OSI & Open System Interconnection \\
CRC & Cyclic Redundancy Check & PCCC & Parallel-Concatenated \\
D8PSK & Differential 8-Phase Shift Keying & PAR & Convolutional Code \\
DBPSK & Differential Binary Phase Shift Keying & PER & Packet Acceptance Rate \\
DQPSK & Differential Quadrature Phase & Phift Keying & PHY Error Rate \\
ECC & Shift Karket & Physical \\
ECS & Error-Correction Code & QoS & Quality of Service \\
EFC & Error Correction Scheme & RA & Repeat Accumulate \\
FEC & Electric Field Communication & RF & Radio Frequency \\
FM & Forward Error Correction & RS & Reed-Solomon \\
GMSK & Frequency Modulation & RSS & Received Signal Strength \\
HARQ & Gaussian Minimum Shift Keying & SPA & Sum-Product Algorithm \\
HBC & Hybrid Automatic Repeat Request & SNR & Signal to Noise Ratio \\
IR & Human Body Communication & SIR & Signal to Interference Ratio \\
LDPC & Impulse Radio & Universal Mobile \\
LOS & Low-Density Parity Check & UWB & Telecommunication System \\
LT & Line Of Sight & Ultra-Wide Band \\
& Luby Transform & WBAN & Wireless Body Area Network \\
& & & Weighted Bit Flipping \\
\hline
\end{tabular}

\section{References}

1. Ullah, S.; Higgins, H.; Braem, B.; Latre, B.; Blondia, C.; Moerman, I.; Saleem, S.; Rahman, Z.; Kwak, K.S. A comprehensive survey of wireless body area networks. J. Med. Syst. 2012, 36, 1065-1094. [CrossRef] [PubMed]

2. Jovanov, E.; Milenkovic, A.; Otto, C.; de Groen, P.C. A wireless body area network of intelligent motion sensors for computer assisted physical rehabilitation. J. NeuroEng. Rehabil. 2005, 2, 6. [CrossRef] [PubMed] 
3. Ullah, S.; Khan, P.; Ullah, N.; Saleem, S.; Higgins, H.; Kwak, K.S. A Review of Wireless Body Area Networks for Medical Applications. arXiv, 2010; arXiv:1001.0831. [CrossRef]

4. Movassaghi, S.; Abolhasan, M.; Lipman, J.; Smith, D.; Jamalipour, A. Wireless body area networks: A survey. IEEE Commun. Surv. Tutor. 2014, 16, 1658-1686. [CrossRef]

5. Rangarajan, A. Emerging trends in healthcare adoption of wireless body area networks. Biomed. Instrum. Technol. 2016, 50, 264-276. [CrossRef] [PubMed]

6. Crosby, G.V.; Ghosh, T.; Murimi, R.; Chin, C.A. Wireless body area networks for healthcare: A survey. Int. J. Ad Hoc Sens. Ubiquitous Comput. 2012, 3, 1. [CrossRef]

7. Barakah, D.M.; Ammad-uddin, M. A survey of challenges and applications of wireless body area network (WBAN) and role of a virtual doctor server in existing architecture. In Proceedings of the 2012 Third International Conference on Intelligent Systems, Modelling and Simulation (ISMS), Kota Kinabalu, Malaysia, 8-10 February 2012; pp. 214-219.

8. Peterson, L.L.; Davie, B.S. Computer Networks: A Systems Approach; Elsevier: Amsterdam, The Netherlands, 2007.

9. Wang, C.; Sklar, D.; Johnson, D. Forward error-correction coding. Crosslink 2001, 3, 26-29.

10. Wicker, S.B. Error Control Systems for Digital Communication and Storage; Prentice Hall: Englewood Cliffs, NJ, USA, 1995; Volume 1.

11. Proakis, J. Digital Communications, 4th ed.; McGraw-Hill Google Scholar: New York, NY, USA, 2001.

12. Costello, D.J. Error Control Coding: Fundamentals and Applications; Prentice Hall: Englewood Cliffs, NJ, USA, 1983.

13. Howard, S.L.; Schlegel, C.; Iniewski, K. Error control coding in low-power wireless sensor networks: When is ECC energy-efficient? EURASIP J. Wirel. Commun. Netw. 2006, 2006, 074812. [CrossRef]

14. Hamming, R.W. Error detecting and error correcting codes. Bell Labs Tech. J. 1950, 29, 147-160. [CrossRef]

15. Gallager, R. Low-density parity-check codes. IRE Trans. Inf. Theory 1962, 8, 21-28. [CrossRef]

16. Divsalar, D.; Jin, H.; McEliece, R.J. Coding theorems for "turbo-like" codes. In Proceedings of the 36th Annual Allerton Conference on Communication Control and Computing, Monticello, IL, USA, 23-25 September 1998; pp. 201-210.

17. Hocquenghem, A. Codes correcteurs d'erreurs. Chiffres 1959, 2, 147-156.

18. Bose, R.C.; Ray-Chaudhuri, D.K. On a class of error correcting binary group codes. Inf. Control 1960, 3, 68-79. [CrossRef]

19. Reed, I.S.; Solomon, G. Polynomial codes over certain finite fields. J. Soc. Ind. Appl. Math. 1960, 8, 300-304. [CrossRef]

20. Benedetto, S.; Montorsi, G. Design of parallel concatenated convolutional codes. IEEE Trans. Commun. 1996, 44, 591-600. [CrossRef]

21. Berrou, C.; Glavieux, A.; Thitimajshima, P. Near Shannon limit error-correcting coding and decoding: Turbo-codes. In Proceedings of ICC '93-IEEE International Conference on Communications, Geneva, Switzerland, 23-26 May 1993; pp. 1064-1070.

22. Beutelspacher, A.; Rosenbaum, U. Projective Geometry: From Foundations to Applications; Cambridge University Press: Cambridge, UK, 1998.

23. Feldman, J.; Wainwright, M.J.; Karger, D.R. Using linear programming to decode binary linear codes. IEEE Trans. Inf. Theory 2005, 51, 954-972. [CrossRef]

24. Viterbi, A. Error bounds for convolutional codes and an asymptotically optimum decoding algorithm. IEEE Trans. Inf. Theory 1967, 13, 260-269. [CrossRef]

25. Bahl, L.; Cocke, J.; Jelinek, F.; Raviv, J. Optimal decoding of linear codes for minimizing symbol error rate (corresp.). IEEE Trans. Inf. Theory 1974, 20, 284-287. [CrossRef]

26. Pearl, J. Probabilistic Reasoning in Intelligent Systems: Networks of Plausible Inference; Morgan Kaufmann: Burlington, MA, USA, 2014.

27. Wiberg, N. Codes and Decoding on General Graphs; Linköping University: Linköping, Sweden, 1996.

28. Comroe, R.; Costello, D. ARQ schemes for data transmission in mobile radio systems. IEEE J. Select. Areas Commun. 1984, 2, 472-481. [CrossRef]

29. Proakis, J.; Salehi, M.; Bauch, G. Contemporary Communication Systems Using MATLAB; Nelson Education: Scarborough, ON, Canada, 2012.

30. IEEE 802.15.6-2012. IEEE Standard for Local and Metropolitan Area Networks_Part 15.6: Wireless Body Area Networks; IEEE: New York, NY, USA, 2012. 
31. Ullah, S.; Mohaisen, M.; Alnuem, M.A. A review of IEEE 802.15. 6 MAC, PHY, and security specifications. Int. J. Distrib. Sens. Netw. 2013, 9, 950704. [CrossRef]

32. Yazdandoost, K.; Sayrafian, K. Channel Model for Body Area Network (BAN); IEEE: Piscataway, NJ, USA, 2010.

33. Takizawa, K.; Hamaguchi, K. Design of packet erasure mitigation technique using a digital fountain code for wearable wireless body area networks. In Proceedings of the 2010 Annual International Conference of the Engineering in Medicine and Biology Society (EMBC), Buenos Aires, Argentina, 31 August-4 September 2010; pp. 356-359.

34. Hamada, Y.; Takizawa, K.; Ikegami, T. Highly reliable wireless body area network using error correcting codes. In Proceedings of the Radio and Wireless Symposium (RWS), Santa Clara, CA, USA, 15-18 January 2012; pp. 231-234.

35. Goto, T.; Takabayashi, K.; Kohno, R. An adaptive error control scheme considering various channel conditions and QoS in medical and non-medical data for WBAN. In Proceedings of the 2016 10th International Symposium on Medical Information and Communication Technology (ISMICT), Worcester, MA, USA, 20-23 March 2016; pp. 1-3.

36. Deepak, K.; Babu, A. Performance Evaluation of Forward Error Correction Schemes in Wireless Body Area Networks. In Intelligent Computing and Applications; Springer: New Delhi, India, 2015; pp. 469-478.

37. Khattiya, W.; Timakul, S.; Choomchuay, S. An error control coding in MAC layer for UWB WBAN. In Proceedings of the 2013 IEEE International Conference on Signal Processing, Communication and Computing (ICSPCC), KunMing, China, 5-8 August 2013; pp. 1-5.

38. Takizawa, K.; Watanabe, K.; Kumazawa, M.; Hamada, Y.; Ikegami, T.; Hamaguchi, K. Performance evaluation of wearable wireless body area networks during walking motions in $444.5 \mathrm{MHz}$ and 2450 MHz. In Proceedings of the 2010 Annual International Conference of the Engineering in Medicine and Biology Society (EMBC), Buenos Aires, Argentina, 31 August-4 September 2010; pp. 370-373.

39. Praveena, V.; Kaythry, P.; Kishore, R. Fountain code based error control technique in wireless body area network. In Proceedings of the Wireless Communications, Signal Processing and Networking (WiSPNET), Chennai, India, 22-24 March 2017; pp. 430-434.

40. Kaythry, P.; Kishore, R.; Praveena, V. Energy Efficient Raptor Codes for Error Control in Wireless Body Area Networks. Wirel. Pers. Commun. 2018, 103, 1-19. [CrossRef]

41. Boussaid, A.; Alaoui, N.; Aghzout, O.; Chakkour, Y.; Alejos, A.V.; Falcone, F. Highly efficient error correcting codes for ubiquitous healthcare in Wireless Body Area Networks. In Proceedings of the 2016 International Conference on Electrical and Information Technologies (ICEIT), Tangiers, Morocco, 4-7 May 2016; pp. 225-230.

42. Youssef, A.A.; Abdelhamid, B.; El-Ramly, S.H.; Elattar, H.M.; Ali, H.H. LDPC Decoding Algorithms for Implant to Implant Wireless Body Area Network. IEEE Access 2018, 6, 13200-13212. [CrossRef]

43. Ahmed, E.; Kohno, R. Error control coding and decoding with medical QoS constraints for WBAN end to end connection via UMTS channel. ICT Express 2018, 4, 181-185. [CrossRef]

44. Reusens, E.; Joseph, W.; Latré, B.; Braem, B.; Vermeeren, G.; Tanghe, E.; Martens, L.; Moerman, I.; Blondia, C. Characterization of on-body communication channel and energy efficient topology design for wireless body area networks. IEEE Trans. Inf. Technol. Biomed. 2009, 13, 933-945. [CrossRef] [PubMed]

45. Fort, A.; Ryckaert, J.; Desset, C.; de Doncker, P.; Wambacq, P.; van Biesen, L. Ultra-wideband channel model for communication around the human body. IEEE J. Select. Areas Commun. 2006, 24, 927-933. [CrossRef]

46. Futatsugi, T.; Sugimoto, C.; Kohno, R. A study on HARQ error-controlling scheme for the wireless control of medical equipment. In Proceedings of the 2012 6th International Symposium on Medical Information and Communication Technology (ISMICT), La Jolla, CA, USA, 25-29 March 2012; pp. 1-4.

47. Liu, W.; Yang, Y.; Wang, F.; Wang, E. Error control strategy in ultrasonic Body Area Networks. China Commun. 2016, 13, 244-259.

48. Suzuki, H.; Kohno, R. Performance analysis of hybrid ARQ error-controlling scheme for UWB BAN. In Proceedings of the 2011 5th International Symposium on Medical Information \& Communication Technology (ISMICT), Montreux, Switzerland, 27-30 March 2011; pp. 94-97.

49. Takabayashi, K.; Tanaka, H.; Sugimoto, C.; Kohno, R. An error control scheme with Weldon's ARQ considering various QoS in medical and non-medical uses for wireless BANs. In Proceedings of the 2013 7th International Symposium on Medical Information and Communication Technology (ISMICT), Tokyo, Japan, 6-8 March 2013; pp. 47-51. 
50. Takabayashi, K.; Tanaka, H.; Sugimoto, C.; Kohno, R. Multiplexing and error control scheme with modified hybrid ARQ for body area network employing IEEE 802.15. 6 in UWB-PHY. In Proceedings of the 8th International Conference on Body Area Networks, Boston, MA, USA, 30 Sptember-2 October 2013; pp. 581-587.

51. Takabayashi, K.; Karvonen, H.; Paso, T.; Tanaka, H.; Sugimoto, C.; Kohno, R. Performance evaluation of a QoS-aware error control scheme for multiple-WBAN environment. IEEJ Trans. Electr. Electron. Eng. 2017, 12, S146-S157. [CrossRef]

52. Takabayashi, K.; Karvonen, H.; Paso, T.; Tanaka, H.; Sugimoto, C.; Kohno, R. Energy efficiency evaluation of ECC scheme utilizing decomposable codes in IEEE std 802.15. 6 based WBANs. In Proceedings of the 10th EAI International Conference on Body Area Networks, Sydney, Australia, 28-30 September 2015; pp. 110-115.

53. Takabayashi, K.; Tanaka, H.; Sugimoto, C.; Kohno, R. Performance evaluation of error control scheme in multihop WBAN based on IEEE802. 15.6. In Proceedings of the 2016 International Symposium on Information Theory and Its Applications (ISITA), Monterey, CA, USA, 30 October-2 November 2016; pp. 365-369.

54. Takabayashi, K.; Tanakay, H.; Sugimoto, C.; Kohno, R. Performance analysis of cross-layer approach about error control scheme for WBANs. In Proceedings of the 2017 11th International Symposium on Medical Information and Communication Technology (ISMICT), Lisbon, Portugal, 6-8 February 2017; pp. 1-5.

55. Takabayashi, K.; Tanaka, H.; Sugimoto, C.; Kohno, R. Performance analysis of multiplexing and error control scheme for body area networks. EURASIP J. Wirel. Commun. Netw. 2016, 2016, 70. [CrossRef]

56. Takabayashi, K.; Tanaka, H.; Sugimoto, C.; Kohno, R. Error control scheme using decomposable codes for various QoS in multiple WBAN environment. In Proceedings of the 2015 9th International Symposium on Medical Information and Communication Technology (ISMICT), Kamakura, Japan, 24-26 March 2015; pp. 83-87.

57. Karvonen, H.; Iinatti, J.; Hämäläinen, M. A cross-layer energy efficiency optimization model for WBAN using IR-UWB transceivers. Telecommun. Syst. 2015, 58, 165-177. [CrossRef]

58. Martelli, F.; Verdone, R.; Buratti, C. Link adaptation in IEEE 802.15. 4-based wireless body area networks. In Proceedings of the 2010 IEEE 21st International Symposium on Personal, Indoor and Mobile Radio Communications Workshops (PIMRC Workshops), Istanbul, Turkey, 26-30 September 2010; pp. 117-121.

59. Kadel, R.; Islam, N. Comparison of Channel Models for Wireless Body Area Networks (WBANs). In Proceedings of the Conference on Wireless Sensors (ICWiSe), Langkawi, Malaysia, 20-22 November 2018.

60. Martelli, F.; Verdone, R.; Buratti, C. Link adaptation in wireless body area networks. In Proceedings of the 2011 IEEE 73rd International Symposium on Vehicular Technology Conference (VTC Spring), Istanbul, Turkey, 26-30 September 2010; pp. 1-11.

61. Kadel, R.; Ahmed, K.; Nepal, A. Adaptive error control code implementation framework for software defined wireless sensor network (SDWSN). In Proceedings of the 2017 27th International Telecommunication Networks and Applications Conference (ITNAC), Melbourne, Australia, 22-24 November 2017; pp. 1-6.

62. Luo, T.; Tan, H.-P.; Quek, T.Q. Sensor OpenFlow: Enabling software-defined wireless sensor networks. IEEE Commun. Lett. 2012, 16, 1896-1899. [CrossRef]

63. De Gante, A.; Aslan, M.; Matrawy, A. Smart wireless sensor network management based on software-defined networking. In Proceedings of the 2014 27th Biennial Symposium on Communications (QBSC), Kingston, ON, Canada, 1-4 June 2014; pp. 71-75.

64. De Oliveira, B.T.; Gabriel, L.B.; Margi, C.B. TinySDN: Enabling multiple controllers for software-defined wireless sensor networks. IEEE Lat. Am. Trans. 2015, 13, 3690-3696. [CrossRef]

65. Trevlakis, S.; Boulogeorgos, A.-A.; Karagiannidis, G. Signal Quality Assessment for Transdermal Optical Wireless Communications under Pointing Errors. Technologies 2018, 6, 109. [CrossRef]

(C) 2018 by the authors. Licensee MDPI, Basel, Switzerland. This article is an open access article distributed under the terms and conditions of the Creative Commons Attribution (CC BY) license (http:/ / creativecommons.org/licenses/by/4.0/). 